\title{
The Evaluation of Chemical Processes for Green Chemistry
}

\author{
Yunshen Zhang ${ }^{1, \text { a }}$ \\ ${ }^{1}$ Department of Petrochemical Engineering, Puyang Vocational and Technical College, Puyang, \\ Henan, P. R. China \\ a472108923@qq.com
}

Keywords: Green Chemistry; Chemical Industry; Environmental Protection; Sustainable Development; Evaluation.

\begin{abstract}
Chemical industry is closely related to people' s life and involves all kinds of aspects. With the development of times, the requirements for chemical engineering is becoming more and more high, which requires the greatest reduction of resource consumption and the adverse impact of chemical process on environment. Thus, it can ensure the sustainable development of chemical process and the whole society. This paper mainly discusses the evaluation of chemical processes for green chemistry. In the process of concretely analyzing and evaluating, this paper mainly analyzes from the current study situation of green chemistry, the required principles of green chemistry, the evaluation of chemical processes for green chemistry and the problems that need to pay attention to the chemical process of green chemistry and other aspects.
\end{abstract}

\section{Introduction}

There is more chemistry in the process of chemical process. With the changing of chemical development concept, we shall stress green chemistry in the process of chemical industry, and green chemistry can also be called "clean chemistry" or "environmentally sound chemistry". Green chemistry covers a number of disciplines, such as analytical chemistry, biochemistry, organic synthesis, etc.. At the same time, we shall pay more attention to the application of green chemistry in the process of chemical industry. Therefore, how to evaluate whether the chemical process meets the requirements of green chemistry is the key to the application of green chemistry in the process of chemical industry. The analysis and evaluation of chemical processes for green chemistry are as follows by mainly combining with relevant research reports in this paper:

\section{The analysis of research status for green chemistry}

With the deepening development of chemical process and the study on the development concept of chemical industry for green chemistry, more and more countries have carried out related research on green chemistry have made some progress. In 1996, the United States Environmental Protection Agency set up a "Presidential Green Chemistry Challenge Award". The installation of this award mastered can promote green chemistry workers constantly to work hard, and then the United States published the first textbooks on green chemistry in 2000; In 1999, the British Royal Society found the first International Journal of Green Chemistry in Britain, while in 2000, they set up green chemistry award; In 1999, Australia set up Green Chemistry Challenge Award, which pushed green chemistry to newly historical stage; In our country, the research of green chemistry is earlier, as early as in 1995, the Department of Chemistry of Chinese Academy of Sciences identified the topic of green chemistry, and the determined subject is "Green Chemistry and Technology". Then in 1996, the Chinese Academy of Sciences held a seminar of "Green Chemistry and Technology in the Industrial Production", meantime, they issued "The Academic Report Compilation of Seminar for Green Chemistry and Technology". In 1997, the green technology and development center held a symposium on green chemistry in the school in the University of Science and Technology of China, and it issued corresponding papers for the research of green chemistry. The name is "Some Major Problems of Current Green Technology". In 1998, the International Symposium on Green Chemistry 
held in the Hefei of Anhui, and Sichuan Union University also set up green chemical and technology research center at the same time. In short, in the research of green chemistry, different countries are constantly increasing research, and our country is certainly no exception. Although certain achievements have been made, there is still a long way to go in the research of green chemistry [1].

\section{The principle of green chemistry needs to follow}

Only clearing the principles that green chemistry needs to follow can we better evaluate green chemistry in the process of chemical chemistry, and the principles that green chemistry needs to abide mainly include the following main points: (1) The pollution prevention is prior to pollution control. Green chemical requires to prevent possible pollution in the process of chemical industry rather than try to find ways to governance after the existence of pollution, which emphasizes that pollution prevention is prior to pollution control. (2) The improvement of atomic economy and the synthesis method used in the process of chemistry require that all starting materials should be able to enter final products. (3) We shall carry out the principle of minimization for the use of toxic materials in the process of chemical reaction, and we require the used chemical products to include toxic products in minimum degree meantime [2]. (4) The scientific nature of chemical product design requires the occurrence of chemical reactions or the formation of chemical products in the product to ensure the use of performance based on the maximum degree of reducing the toxicity of product itself. (5) For the use of renewable raw materials, if technology is feasible and economy is reasonable in the process of chemical reaction, it should be based on renewable raw materials in the chemical reaction. (6) The non-toxic harmless of solvents and additives also require a certain amount of solvents and additives in the process of chemical reaction. For the situation where has to use, we need to ensure the non-toxic harmless of solvent and auxiliary. (7) The minimization of energy consumption in chemical synthesis needs to meet certain temperature and pressure conditions in the process of chemical synthesis. Besides, it needs to carry out effective design for chemical reaction to ensure that temperature and pressure is in the best condition in chemical reaction. (8) For the simplify in the process of chemical reaction, partial biochemical steps may abolish this kind of derivative process without affecting the basis of product performance in the process of chemical reaction, thereby simplifying reaction process, reducing raw materials, energy consumption and capital investment [3]. (9) For the choice of catalyst, catalyst plays an important role in the process of chemical reaction. In accordance with the requirements of green chemistry, the catalyst should be based on highly selective catalysts. (10) After using the degradation of chemical products, chemical reactions and the products that forms in the process of chemical industry after chemical synthesis for a certain number of years, these products can be explained, and degradation products meet the requirements of non-toxic harmless, which achieves the recycling of resources and reduces the impact on environment. (11) The application of chemical analysis technology in chemical synthesis needs to pay attention to the application of chemical analysis technology in the process of chemical synthesis in order to facilitate the implementation of actual monitoring in the field of chemical synthesis and to avoid the formation and generation of hazardous substances in intermediate links. (12) The safety in the process of chemical synthesis has certain security risks in the process of chemical synthesis, which is easy to have fire, explosion and other safety incidents. The above 12 principles are the principles of green chemistry that needs to be followed, and it also the basis for the application evaluation of green chemical in the process of chemical industry meanwhile. If the chemical process does not meet the above principles, the corresponding chemical process will not meet the requirements of green chemistry.

\section{The evaluation and analysis of chemical process for green chemistry}

Atomic economy evaluation index. In the process of chemical industry, certain evaluation system is needed to evaluate whether the chemical process meets green chemistry. In the green chemistry evaluation index, in the early 90s of last century, the United States Stanford University professor Tost B.M put forward the evaluation index of atomic economy. This index emphasizes the use efficiency of raw materials in chemical process, and the corresponding formula is: 
(Expected product molecular weight / sum of atomic weights of all reactants) x $100.0 \%$.

The calculation formula is mainly to measure the important indicators of the conversion from reactants to products. If all the reactants enter into final products in the reactants, the atom economy can reach $100.0 \%$. Of course, this is an ideal state, in the process of infinite close to an ideal state, we need to take measures to ensure chemical process to meet the requirements of green chemistry. For example, there is no by-products' formation in the products, the whole reaction does not use protective groups, etc.The indicators are too idealized in general, which ignores some special circumstances the whole chemical process, such as: product yield, reagent overdose, the loss amount of solvent in the reaction, the too much amount of added reactants, etc. Therefore, the atomic economy has certain biases in the process of evaluating green chemistry, but it also needs to combine other corresponding indicators [4].

The evaluation index of environmental factors. With the deepening of research, some scholars have put forward the concept of environmental factors, and this indicator emphasizes the waste in the process of chemical synthesis, and the using formula is:

Environmental Factor $=$ Waste $/$ Expected Product

The unit of waste and expected product is $\mathrm{Kg}$. That is, it is corresponding to $1 \mathrm{Kg}$ of the output of waste in the process of expected products. The waste of indicator refers to expected products other than all by-products. The corresponding influence on environment is greater, and the environmental factors under ideal conditions are 0 , that is, there is not the existence of waste in the production of expected products. Of course, in the actual chemical process, it is impossible that waste output is zero, it focus on the amount of waste in the same environmental factors, while the toxicity of waste and so on are not considered. Therefore, environmental factors cannot truly reflect the impact of chemical process on the environment, and actual green chemical evaluation also needs to refer to other indicators, such as the follow-up scholars proposed life cycle assessment indicators, etc.

The index analysis of solution green chemical evaluation. Because most chemical reaction is carried out in solution, and the evaluation of green chemistry can also be analyzed from relevant indicators solution. Chemical reaction occurs in solution can also refer to some indicators, and they will be applied to the evaluation of green chemistry, and specific indicators are:

For the chemical reaction in solution, in carrying out the evaluation project of green chemistry, in addition to taking reactants and final product into account, we should also analyze the green degree of whole reaction process by some external factors.

In accordance with the concept of the amount of solution fluctuations, the residue into pure water after process needs to go through more steps after chemical reaction, and this step is Px. If the Px is greater, the residual liquid into pure water process needs more steps, and the corresponding amount of solution fluctuations is greater, and the performance of residue has greater impact on environment. This can process the corresponding steps in the original reaction process and reduce Px so as to ensure the maximum degree of green in the chemical reaction.

The application of solution fluctuations can reflect the friendly degree of chemical reaction to environment from another angle. By analyzing the fluctuation of solution in later stage, it is possible to identify the impurity in the residual liquid or insoluble ion, so that the original chemical reaction process can optimize design in order to achieve continuous improvement in the process of chemical reaction. For the different chemical processes involved in solution, it can use the fluctuation of solution to carry out assessment to achieve the improvement of chemical reaction and reduce the impact on environment. It can also realize the maximization application of raw materials, energy and capital investment and improve the green degree of green chemistry in the greatest degree [5].

\section{The problems that need to pay attention in chemical process of green chemistry}

Chemical production is in line with the requirements of green chemistry in the greatest degree, and we need to note the following points in actual chemical process: (1) It emphasizes pollution prevention before the process of chemical industry, for a long time, many chemical companies are used to pollute in chemical process first and then treat. Therefore, after the green chemical evaluation 
of chemical process, the chemical reaction line of part optimal design emerge into it. (2) Pay attention to the selection of raw materials in chemical process, and chemical raw materials are also key in green chemistry. In chemical process, renewable resources can replace non-renewable resources, and the raw material itself needs to have the requirements of high efficiency and low pollution. (3) For the concept of green of comprehensive and in-depth understanding, the in-depth understanding of green chemistry is to ensure that the foundation of evaluation of chemical process for green chemistry. The waste is least after the completion reaction, and it has less impact on environment to ensure the atomic utilization of chemical reaction and to ensure that the final chemical products are conducive to environmental protection and have no adverse effects on human body.

\section{Concluding remarks}

The suggestion of green chemistry can evaluate and analyze the adverse effects of chemical process, so as to control adverse effects. At this stage, it requires the combination of a variety of evaluation methods in the evaluation of green chemistry to carry out comprehensive evaluation for the chemical process of green chemistry.

\section{References}

[1] Huo Zepeng. Discussion on Green Chemistry and the Quantitative Assessment of Green Degree of Chemical Products and Chemical Processes [J]. Shandong Chemical Industry, 2015 (20).

[2] Wang Huanxin. An Analysis of Green Chemistry Has Become the Subject of Twenty-first Century [J]. Guangzhou chemical industry, 2011 (19).

[3] Zheng Huiqin, Li Chengwei, Li Ke, et al. The Application of Green Process Engineering in Chemical Industry and Chemical Experiment Teaching [J]. Journal of Henan Institute of Education (Natural Science Edition), 2011 (04).

[4] Long Mingyuan, Wang Yuanyuan, Yang Guoqing. Discussion on Sustainability of Green Chemistry and Pharmaceutical R \& D and Production [J]. Chemical Industry Management, 2013 (08).

[5] Cao Wanwan. The Study of Related Issues for Green Chemistry and Chemical Industry [J]. The Technical Development of Enterprises, 2014 (17). 